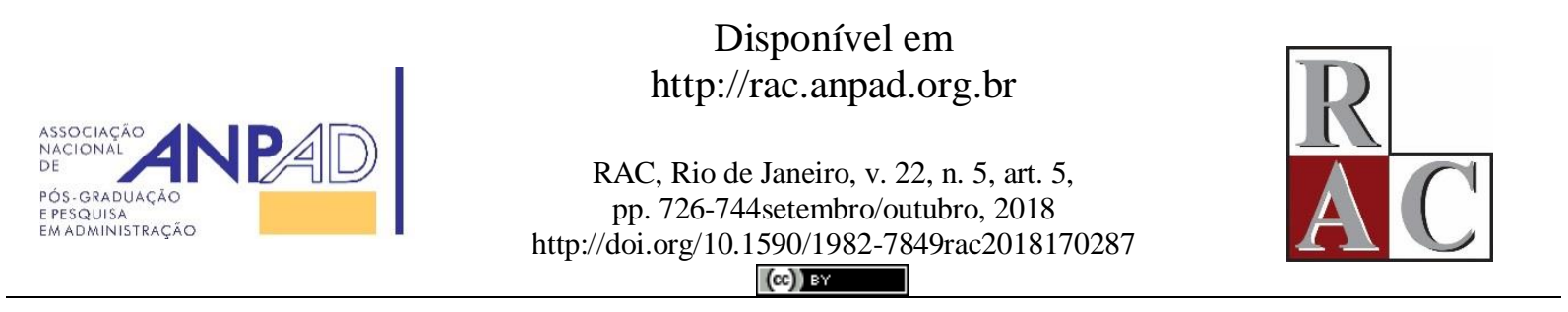

\title{
Desenvolvimento de um Framework de Adoção da Inovação no Mercado de Baixa Renda
}

\section{Development of an Innovation Adoption Framework in a Low-Income Market}

\author{
Vitor Koki da Costa Nogami ${ }^{1}$ \\ https://orcid.org/0000-0001-5185-731X \\ Andres Rodriguez Veloso ${ }^{2}$ \\ https://orcid.org/0000-0003-3037-6773
}

Universidade Estadual de Maringá, Departamento de Administração, Maringá, PR, Brasil ${ }^{1}$ Universidade de São Paulo, Faculdade de Economia, Administração e Contabilidade, São Paulo, SP, Brasil ${ }^{2}$ 


\title{
Resumo
}

Pesquisas sobre a inovação e o mercado de baixa renda têm focado eminentemente no desenvolvimento de produtos e gestão de marketing, assumindo uma postura top-down das empresas para os consumidores. Do ponto de vista do indivíduo, tem sido dada pouca atenção para compreender a adoção da inovação pelos consumidores de baixa renda. O presente artigo tem como objetivo apresentar o desenvolvimento de um framework de adoção da inovação do consumidor de baixa, renda assumindo uma postura bottom-up, focando principalmente nos consumidores e em suas restrições estruturais e individuais encontradas no contexto de pobreza. Os pressupostos teóricos que embasam o framework consistem nas restrições encontradas pelos consumidores do mercado da base da pirâmide, que englobam baixa renda, falta de informação, conhecimento e confiança. Para buscar alternativas a fim de minimizar esse efeito, procurou-se na literatura fatores que pudessem influenciá-lo. Como resultado, o artigo apresenta um framework teórico que busca evidenciar a relação entre as restrições da baixa renda e sua influência na adoção da inovação. Partindo do pressuposto de que essa é uma relação negativa, o framework apresenta variáveis sustentadas na literatura de inovação e baixa renda, que podem influenciar esse efeito principal.

Palavras-chave: adoção da inovação; inovação; base da pirâmide; baixa renda.

\begin{abstract}
Research on innovation and the low-income market has focused primarily on product development and marketing management, taking a top-down approach, from business to consumer. From the individual's point of view, little attention has been paid to understanding the adoption of innovation by low-income consumers. This article aims to present the development of a framework for innovation adoption by low-income consumers assuming a bottomup approach, focusing mainly on consumers and their structural and individual constraints found in the context of poverty. The theoretical assumptions underlying the framework are the constraints faced by market consumers at the base of the pyramid, which include low income, lack of information, knowledge and confidence. In order to find alternatives to minimize this effect, we searched in the literature for factors that could influence this effect. As a result, the paper presents a theoretical framework that seeks to show the relationship between the constraints in the low-income market and its influence on innovation adoption. Based on the supposition this is a negative relationship, the framework presents variables supported by literature on innovation and low-income markets, which can influence this main effect.
\end{abstract}

Keywords: innovation adoption; innovation; base of the pyramid; low-income.

JEL codes: I31, I31, O35. 


\section{Introdução}

Do ponto de vista da gestão de marketing, a inovação é frequentemente reconhecida como um dos fatores críticos de sucesso das empresas multinacionais (Multinational Companies [MNC]). O conceito de inovação é costumeiramente remetido a elementos de tecnologia, tendo como origem países desenvolvidos onde as principais empresas multinacionais estão localizadas (Govindarajan \& Ramamurti, 2011; Hart \& Christensen, 2002; Prahalad, 2005; Ramani \& Mukherjee, 2014; Viswanathan, Shultz, \& Sridharan, 2014). Os custos de produção e P\&D encarecem os custos dos produtos, refletindo em altos preços para o consumidor final, tornando a inovação acessível apenas para consumidores que têm alto poder aquisitivo (Corsi \& Di Minin, 2014; Prahalad, 2012). Pretendemos, com este artigo, apresentar uma lógica inversa, na qual é possível desenvolver e adotar a inovação em mercados emergentes. Em outras palavras, adotamos uma abordagem bottom-up em vez da tradicional abordagem top-down (Viswanathan, Yassine, \& Clarke, 2011), comumente utilizada pela maioria das empresas e encontrada em muitas publicações sobre a inovação e o mercado de baixa renda.

Não é suficiente estudar a inovação só do ponto de vista da tecnologia, da modernidade e da sofisticação. É necessário, também, estudar a inovação do ponto de vista social e conjuntural (Nogami, Vieira, \& Veloso, 2018). É comum e intuitivo assumir que tradicionalmente os consumidores de baixa renda não adotam produtos inovadores facilmente, tendo em vista suas restrições individuais de renda, conhecimento e informações causadas por falta de condições estruturais de educação, saúde, moradia e transporte, que são comuns em ambientes de baixa renda (Kolk, Rivera-Santos, \& Rufin 2014; Nakata \& Weidner, 2012; Viswanathan, 2016; Viswanathan et al., 2011). Nossa proposta é ir contra essa visão intuitiva, apontando que a adoção da inovação (consumer innovativeness) no mercado da camada mais pobre da população, chamada de base da pirâmide (Bottom of the Pyramide [BOP]), é possível e viável. Servir apenas aos consumidores do topo da pirâmide é uma forma de miopia tanto de empresas em países desenvolvidos quanto de empresas em países emergentes (Corsi \& Di Minin, 2014; Faria \& Hemais, 2017; Yurdakul, Atik, \& Dholakia, 2017).

As empresas estão identificando e aproveitando a oportunidade de ofertar bens e serviços aos consumidores de baixa renda que vivem na pobreza em mercados emergentes (Hemais, Borelli, Casotti \& Dias, 2014; Ray \& Ray, 2011; Subramaniam, Ernst, \& Dubiel, 2015). A base da pirâmide constitui o maior mercado global restante para as empresas captarem novos clientes. Até recentemente, esse segmento vinha sendo ignorado devido à sua falta de atrativos aparente e de desafios não rentáveis em comparação com os consumidores de renda média e alta (Nakata \& Weidner, 2012). Porém, com o crescimento econômico, os consumidores da BOP são capazes de pagar por produtos de qualidade adaptados às suas necessidades (Barki \& Parente, 2010). A BOP é capaz de oferecer grande potencial de mercado com benefícios expressivos, como crescimento econômico, lucro e contribuições ambientais e sociais para a humanidade.

As organizações estão buscando entrar nesse segmento por diversos mercados, contudo, a adoção de novos produtos é um dilema para qualquer consumidor. No contexto da baixa renda, existem algumas barreiras estruturais que tornam esse processo de inovação mais lento. Tais barreiras devem ser compreendidas para acelerar o processo de adoção de inovação por esses consumidores (Nakata \& Weidner, 2012). De acordo com Khanna e Palepu (2013), existem barreiras estruturais no contexto da BOP, que geralmente são compostos por restrições de infraestrutura. Problemas com transporte, educação deficitária, poucos cuidados com a saúde, dificuldade de acesso à energia e ao saneamento são situações comuns em regiões mais pobres (Venugopal \& Viswanathan, 2017; Viswanathan, 2016; Weidner, Rosa, \& Viswanathan, 2010).

Diante desse cenário que envolve um contexto de restrições estruturais em ambientes de pobreza, a adoção da inovação pode ser potencializada em busca de melhorias de qualidade de vida. Tendo em vista o interesse das organizações em difundir suas tecnologias, o poder de compra relativo que se mostrou crescente nos últimos anos e o interesse das pessoas em se inserir em um mercado com maiores inovações, o presente artigo tem como objetivo desenvolver um framework de adoção da 
inovação considerando as restrições encontradas no contexto da baixa renda. Para tanto, são revisados diferentes conceitos encontrados na literatura que envolvam a relação entre inovação e baixa renda, a saber: UTAUT2, 4 As de Anderson e Billou, 12 Princípios de Prahalad, Inovação Disruptiva, Inovação Reversa e Inovação Inclusiva.

\section{Fundamentação Teórica}

A sustentação teórica para elaboração do framework consiste na revisão de oito principais conceitos. Inicialmente, apresentamos a literatura sobre baixa renda, que aponta as restrições do consumidor da BOP, as quais os impedem de adotar inovações (Nakata \& Weidner, 2012). Depois, são apresentados os conceitos de adoção da inovação com base na literatura de consumer innovativeness (Midgley \& Dowling, 1978). Esses dois primeiros conceitos compõem a relação direta (efeito principal) do framework. Em seguida, são apresentados os conceitos que desempenham o papel de variáveis influenciadoras nesse efeito principal.

\section{Restrições da BOP}

Muito se fala sobre o tamanho e o potencial de consumo do mercado de baixa renda, no entanto, do ponto de vista acadêmico de Marketing e Inovação, pouco se tem explorado de maneira contundente as reais condições de incerteza e problemas estruturais presentes em ambientes de pobreza. Regiões suburbanas, favelas e vilarejos rurais têm muitas restrições estruturais que envolvem dificuldades de moradia, transporte e educação, bem como restrições individuais de renda, informação, conhecimento e confiança.

De acordo com o relatório Next 4 Billion, do World Resources Institute (Hammond, Kramer, Katz, Tran, \& Walker, 2007), os consumidores da BOP são penalizados pelas condições de pobreza com altos preços pelos bens e serviços (Kaplinsky et al., 2009; Mendoza, 2011). Esse conceito também é conhecido por poverty penalty. Para Mendoza (2011), poverty penalty, ou penalização da pobreza, refere-se aos preços relativamente mais altos pagos pelas pessoas que vivem na pobreza quando comparados com as pessoas não pobres, em determinados mercados. Essa penalização se manifesta por preços mais altos, qualidade e desempenho inferiores dos produtos, falta de acessibilidade no mercado e infraestrutura defasada em termos de saúde, transporte e educação (Mendoza, 2011).

Essa penalização pela pobreza acontece também no acesso a crédito, nos cuidados com a saúde, na rede de energia e no saneamento. O crédito tanto para consumo quanto para empreender é caro pelo fato da instabilidade econômica das pessoas, pela falta de qualificação para gestão, entre outros problemas estruturais (Kaplinsky et al., 2009; Mendoza, 2011). Sem esse auxílio de financiamento, os consumidores não podem fazer aquisições caras e os empreendedores não podem investir em seus negócios, restringindo o potencial econômico do mercado. Além da renda limitada, outro fator que ajuda a compor o orçamento familiar são os trabalhos informais e os temporários (Abdelnour \& Branzei, 2010), conhecidos como bicos. A falta de estabilidade de renda impede que a BOP faça um planejamento financeiro tanto em curto quanto em longo prazo.

Outro fator que restringe as habilidades no mercado da BOP é o analfabetismo, completo ou funcional, que é presente em comunidades desfavorecidas, tornando a relação com as compras prejudicada (Weidner et al., 2010). Para serem consideradas alfabetizadas funcionalmente, as pessoas devem saber ler e compreender o conteúdo e as mensagens escritas, bem como realizar contas matemáticas para estarem aptas aos cálculos cotidianos que envolvem juros, impostos, empréstimos, financiamentos e relação com as compras. $\mathrm{O}$ índice de analfabetismo completo ou funcional no segmento de baixa renda é presente, acarretando em dificuldades do dia a dia (Parente, Limeira, \& Barki, 2008).

Além do analfabetismo, a falta de informação é presente nesse contexto (Viswanathan \& Rosa, 2010). O restrito acesso à internet e a dificuldade de leitura de textos, muitas vezes formais demais, 
tornam a informação mais limitada ao consumidor (Abdelnour \& Branzei, 2010). No sentido da falta de informação, o consumidor de baixa renda também é desprovido de conhecimento acerca da tecnologia (Hang, Chen, \& Subramian, 2010; Nakata \& Weidner, 2012). Em muitos casos, os produtos tecnológicos exigem conhecimento prévio e avançado sobre recursos, dispositivos e acessórios de uso.

As penalizações, o analfabetismo e a falta de informação geram condições difíceis para a vida dos consumidores que vivem em condições de pobreza (Weidner et al., 2010), acarretando em problema de moradia e saúde pública (Viswanathan \& Sridharan, 2012). Atrelados aos problemas de infraestrutura, essas restrições refletem em más condições de transporte, comunicação, saneamento, alimentação, eletricidade e educação (Hang et al., 2010; Nakata \& Weidner, 2012). Todas essas restrições tornam o consumidor mais inseguro e menos confiante para adotar inovações (Viswanathan \& Sridharan, 2012).

A inovação no mercado da base da pirâmide não acontece da mesma maneira que no mercado do topo da pirâmide. O conceito de vazios institucionais (institutional voids) apresentado por Khanna e Palepu (2013) explora as condições prejudicadas de infraestrutura encontradas nos mercados menos desenvolvidos e fornece orientação sobre como as organizações devem mudar suas escolhas estratégicas para alcançar o mercado de baixa renda. As restrições em termos de falta de eletricidade, atraso no transporte, questões básicas de alimentação, higiene, habitação, saúde, baixa escolaridade, falta de conhecimento e informação e grande instabilidade econômica são incertezas no mercado de baixa renda - inovar sob essas condições de incerteza requer diferentes diretrizes.

Desenvolver o mercado na BOP é uma forma de diminuir os problemas econômicos de países em desenvolvimento (Viswanathan \& Rosa, 2010). As organizações que entram nesse segmento, não só para vender mas também para fomentar negócios, podem atender a essa demanda reprimida e contribuir para a melhoria de vida dessas pessoas, ajudando na redução da pobreza (Nogami et al., 2018). Com base nesse argumento, os países em desenvolvimento são os mercados-alvo ideais para o desenvolvimento de inovações. Em outras palavras, há relação entre inovação e o mercado de baixa renda.

\section{Adoção da inovação (Consumer innovativeness)}

Entre os trabalhos clássicos, Midgley e Dowling (1978) e Hirschman (1980) desenvolveram conceitos de innovativeness e ligaram com a adoção de novos produtos. Esses trabalhos foram precursores para a evolução do constructo no que tange ao comportamento do consumidor (Manning, Bearden, \& Madden, 1995). Midgley e Dowling (1978) definiram capacidade inata de inovação (innate innovativeness) como o grau que o indivíduo toma decisões sobre adoção da inovação, independentemente das opiniões de outras pessoas. O conceito de capacidade de inovação de Hirschman (1980) foca no desejo dos consumidores em obter informações sobre aquela inovação, sendo considerada como a inerente busca por novidade e é definida como o desejo de buscar o novo e o diferente.

Os pesquisadores de consumo referem-se ao conceito de Midgley e Dowling (1978) como a capacidade de fazer julgamentos de forma independente; já o conceito de Hirschman (1980) é considerado como capacidade de fazer buscas inerentes (Manning et al., 1995). Tendo em vista a dificuldade de operacionalização desses conceitos, são sugeridas formas de mensurar tais constructos para identificar as características dos consumidores que são mais propensos a adotarem a inovação e, então, desenvolver uma medida correspondente para a definição de capacidade de innovativeness. Tendo em vista esse contexto, Manning, Bearden e Madden (1995) desenvolveram uma escala para mensurar consumer innovativeness com base nesses dois conceitos.

O primeiro constructo, desenvolvido com base nas ideias de Midgley e Dowling (1978), é o Consumer Independent Judgment Making (CIJM), que é a capacidade de o consumidor fazer julgamentos, independentemente de outras pessoas, a respeito de uma inovação. Em outras palavras, é a medida da tendência do consumidor em tomar decisões sem levar em consideração a opinião de terceiros. O segundo constructo, sustentado por Hirschman (1980), é a busca inerente de novidades para comportamentos de consumo de novos produtos, definido como Consumer Novelty Seeking (CNS), que 
é a procura do consumidor por novidades. Essa construção representa a motivação dos consumidores para obter informações a respeito de novos produtos a partir de fontes que incluem principalmente a comunicação de marketing.

Ao associar a literatura sobre o mercado de baixa renda na seção anterior com a literatura sobre adoção da inovação na presente seção, é possível introduzir a primeira proposição deste estudo, que é o efeito principal do framework conceitual proposto.

Proposição 1. As restrições contextuais da BOP influenciam negativamente na adoção da inovação pelos consumidores.

Tendo em vista essa relação direta entre as restrições da BOP e a adoção da inovação, são apresentados em seguida os conceitos de inovação que envolvem o contexto da baixa renda. Considerando as peculiaridades desse mercado, no qual há mais dificuldades tanto do ponto de vista individual (renda, confiança, informação) quanto do ponto de vista coletivo (infraestrutura, emprego, preconceito), a literatura revisada para a construção do framework tem como pressuposto considerar esse contexto de incerteza.

\section{Antecedentes do UTAUT 2}

Inicialmente, o modelo Theory of Reasoned Action (TRA), desenvolvido por Fishbein e Ajzen (1975), era um consolidado modelo para prever e explicar comportamentos; porém, de forma geral, não especificamente no campo da adoção de inovações tecnológicas. Então Davis, Bagozzi e Warshaw (1989) desenvolveram, com base no TRA, o Technology Acceptance Model (TAM), que inclui a aceitação da tecnologia para pesquisar especificamente o uso de computadores, tornando o modelo mais restrito e objetivo para esses casos.

O modelo TAM apresenta duas variáveis principais: facilidade percebida de uso (perceived ease of use) e utilidade percebida (perceived usefulness), que determinam a atitude e o comportamento dos usuários para a aceitação de novas tecnologias. Facilidade percebida de uso é definida como o grau em que a crença da pessoa que usa um sistema particular seria livre de esforço, a utilidade percebida é o grau em que uma pessoa entende que o uso de um sistema particular aumentaria o desempenho do seu trabalho (Davis, Bagozzi, \& Warshaw, 1989).

Venkatesh, Morris, Davis, e Davis (2003), com base em TRA, TAM e em outros modelos que buscam compreender e explicar comportamentos (Motivational Model, Theory of Planned Behavior, Model of PC User, Innovation Diffusion Theory e Social Cognitive Theory), desenvolveram o Unified Theory of Acceptance and Usage of Technology (UTAUT), utilizado para explicar a aceitação da tecnologia da informação pelo usuário em diversas áreas. O UTAUT é um dos modelos mais completos, uma vez que leva em consideração vários modelos anteriores. Nove anos depois da publicação do UTAUT, Venkatesh, Thong e Xu (2012) desenvolveram o UTAUT2, que inclui como antecedentes os constructos motivação hedônica, preço e hábito.

O que todos os modelos têm em comum é o objetivo de explicar quais constructos antecedem o comportamento e o uso de novas tecnologias. Com o surgimento de muitos produtos tecnológicos acessíveis ao dia a dia do consumidor a partir dos anos 1990, conhecer os fatores que influenciam o uso e o não uso desses dispositivos se tornou interesse da academia e do mercado, por isso o avanço no desenvolvimento desses modelos. O modelo UTAUT 2 tem sete constructos antecedentes tanto da intenção quanto do comportamento de aceitação e uso de tecnologia que se enquadram como variáveis influenciadoras do efeito principal apresentado, ou seja, são fatores que podem melhorar a relação negativa entre as restrições da BOP e a adoção da inovação (proposição 1). A Tabela 1 apresenta os constructos do UTAUT2. 
Tabela 1

Constructos do UTAUT 2

\begin{tabular}{ll}
\hline $\begin{array}{l}\text { Expectativa de } \\
\text { Desempenho }\end{array}$ & $\begin{array}{l}\text { É o nível que o consumidor acredita que o uso da inovação vai ajudá-lo a alcançar em } \\
\text { benefícios no desempenho do trabalho, ou seja, tem fins funcionais. Esse constructo é } \\
\text { composto pela utilidade percebida, motivação extrínseca, ajuste do trabalho, vantagem } \\
\text { relativa e resultados esperados. }\end{array}$ \\
\hline $\begin{array}{l}\text { Expectativa do } \\
\text { Esforço }\end{array}$ & $\begin{array}{l}\text { É o nível em que o consumidor acredita que terá que exercer de esforço para adotar a } \\
\text { inovação e, então, ter os benefícios recebidos. Esse constructo é composto pela percepção } \\
\text { de facilidade de uso, complexidade e pela própria facilidade de uso. }\end{array}$ \\
\hline Influência Social & $\begin{array}{l}\text { É definida como o nível em que o consumidor percebe a importância das crenças de outros } \\
\text { indivíduos para avaliar a inovação. Esse constructo é composto pelas normas subjetivas, } \\
\text { pelos fatores sociais e pela imagem. }\end{array}$ \\
\hline Condições & $\begin{array}{l}\text { É o nível em que o indivíduo acredita que existe infraestrutura organizacional e técnica } \\
\text { para suportar o uso da inovação. Esse constructo é composto pelo controle do } \\
\text { Facilitadoras }\end{array}$ \\
\hline comportamento percebido, condições facilitadoras e compatibilidade. \\
\hline Hedônação & $\begin{array}{l}\text { É o prazer percebido pelo usuário ao adotar inovação, ou seja, diversão, alegria, } \\
\text { divertimento, entretenimento e prazer. }\end{array}$ \\
\hline Preço & $\begin{array}{l}\text { É o nível com que o valor monetário vai influenciar na adoção da inovação. A lógica é a } \\
\text { mesma do valor percebido quando o valor é a relação do somatório dos benefícios em } \\
\text { relação ao somatório dos sacrifícios, porém, apenas em relação aos sacrifícios monetários. }\end{array}$ \\
\hline Hábito & $\begin{array}{l}\text { É representado pela experiência, ou seja, o hábito de usar ou estar em contato com } \\
\text { elementos correlatos com a inovação aumenta a probabilidade de o consumidor adotar a } \\
\text { inovação. }\end{array}$ \\
\hline
\end{tabular}

Nota. Fonte: Elaborado pelos autores com base em Venkatesh, V., Morris, M. G., Davis, G. B., \& Davis, F. D. (2003). User acceptance of Information technology: Toward a unified view (p. 447). MIS Quarterly, 27(3), 425-478. https://doi.org/10.2307/30036540 e Venkatesh, V., Thong, J. Y., \& Xu, X. (2012). Consumer acceptance and use of information technology: Extending the unified theory of acceptance and use of technology (p. 160). MIS Quarterly, 36(1), 157-178. https://doi.org/10.2307/41410412

Considerando os elementos que compõem o modelo UTATUT2 que podem ser acrescentados ao framework conceitual do nosso artigo, é possível apresentar a proposição 2, que introduz esses elementos como variáveis adicionais.

Proposição 2. A relação entre as restrições da BOP e a adoção da inovação é positivamente influenciada pelos antecedentes do UTAUT2 - (P2a) Expectativa de Desempenho, (P2b) Expectativa de Esforço, (P2c) Influência Social, (P2d) Condições Facilitadoras, (P2e) Motivação Hedônica, (P2f) Preço e (P2g) Hábito.

\section{Quatro As de Anderson e Billou}

As empresas ainda lutam para entender os fatores críticos de sucesso no mercado da BOP e alcançar rentabilidade e sustentabilidade organizacional, especificamente em termos de inovação. Uma questão-chave para as MNCs é como ativar a inovação nesses mercados e aproveitar o seu tamanho e o seu potencial econômico (Anderson \& Billou, 2007). Não por acaso, tendo em vista essa oportunidade, existem diversos trabalhos acadêmicos e não acadêmicos com objetivo de prescrever tarefas e procedimentos para gestão de marketing. Trabalhos que relacionam a inovação nesse contexto também ganham força na academia (Subramaniam et al., 2015).

Nesse sentido, para obter sucesso no mercado da BOP, as empresas devem desenvolver modelos de negócios específicos para as necessidades e os desafios dessa população (Hang et al., 2010; Prahalad, 2005). As ações locais no ambiente de baixa renda são determinantes para o sucesso das empresas (Govindarajan \& Ramamurti, 2011; Viswanathan \& Sridharan, 2012). Esses novos modelos de negócios 
têm que estar adequados ao contexto da baixa renda; desse modo, melhorar o acesso, disponibilizar facilidade de pagamento, apresentar soluções locais e adaptar os negócios para atender esse consumidor são meios para obter sucesso na BOP (Foster \& Heeks, 2014). Com base nessas diretrizes, Anderson e Billou (2007) apontam que, para ofertar inovação de maneira eficaz no mercado da BOP são necessários quatro fatores, os 4 As: affordability (acessibilidade), acceptability (aceitabilidade), availability (disponibilidade) e awareness (consciência).

Acessibilidade (affordability) é o grau em que os bens e os serviços de uma empresa são acessíveis aos consumidores da BOP do ponto de vista financeiro, sendo fundamental para alcançar as pessoas com baixa renda que, até então, eram não consumidores (Anderson \& Billou, 2007; Barki \& Parente, 2010). Muitas pessoas desse segmento vivem com salários diários, semanais, ou seja, pontuais, conhecidos como bicos realizados de forma muitas vezes inconstante (Nogami, Carvalho, Toledo, Veloso, \& Gouvêa, 2015). As empresas precisam entregar inovações que caibam dentro dessa limitação, não só da quantidade de renda, mas dessa despadronização e da inconstância salarial. Essa é uma restrição que, em muitos casos, impossibilita a aquisição de empréstimo. Assim, fornecer acessibilidade contribui para encorajar a adoção da inovação no mercado da BOP (Prahalad, 2012).

Aceitabilidade (acceptability) é o nível que os consumidores e os outros agentes da cadeia de valor estão dispostos a consumir, produzir, fornecer ou vender determinado produto ou serviço (Anderson \& Billou, 2007). Em mercados nos quais os consumidores têm recursos limitados, as empresas inovadoras mais bem-sucedidas criam produtos e serviços que são adaptados às necessidades específicas de clientes, distribuidores ou ambos (Anderson \& Markides, 2007). As empresas devem atentar para as questões culturais, sociais e regionais para atender às necessidades específicas da BOP e aumentar a sua aceitação (Prahalad, 2005). Assim, proporcionar aceitabilidade contribui para encorajar a adoção da inovação no mercado da BOP.

Disponibilidade (availability) é o grau que sinaliza que os clientes são capazes de adquirir e usar um produto ou serviço (Prahalad, 2012). Os canais de distribuição nesses mercados emergentes são comumente fragmentados ou até mesmo inexistentes, tornando a distribuição um desafio, tendo em vista as barreiras encontradas no ambiente da BOP (Anderson \& Billou, 2007). Regiões rurais da China e da Índia e em grandes favelas nas regiões metropolitanas brasileiras são locais onde o acesso não é fácil e a distribuição das empresas encontra dificuldade para atuar. Assim, proporcionar canais de distribuição para tornar o produto disponível contribui para aumentar a adoção da inovação no mercado da BOP (Prahalad, 2005).

Consciência (awareness) se refere sobre o que os consumidores sabem acerca dos produtos e serviços que a empresa vende (Anderson \& Markides, 2007). Uma vez que muitos indivíduos não têm acesso às propagandas e às mídias, a construção de consciência é um desafio. Portanto, as empresas devem desenvolver formas alternativas para comunicar sua oferta, bem como buscar proporcionar que a mídias convencionais cheguem até o consumidor. Assim, a consciência do produto encoraja a adoção da inovação no mercado da BOP (Anderson \& Billou, 2007; Prahalad, 2012).

Portanto, tendo em vista essas facilidades que as empresas precisam proporcionar ao consumidor para encorajar a adoção da inovação, é possível apresentar nossa Proposição 3, que incluem os 4 As de Anderson e Billou como variáveis influenciadoras do efeito principal do framework:

Proposição 3: A relação entre as restrições da BOP e a adoção da inovação é positivamente influenciada pelos 4 As de Anderson e Billou (2007) - (P3a) Acessibilidade, (P3b) Aceitabilidade, (P3c) Disponibilidade e (P3d) Consciência.

\section{Doze princípios da inovação de Prahalad}

Para Prahalad (2005), é necessária uma nova filosofia para o desenvolvimento de produtos no mercado da BOP. Essa filosofia é diferente das diretrizes encontradas nas empresas ocidentais. Com base em suas pesquisas científicas, pesquisas de mercado e experiência profissional, o autor identificou 12 princípios que, em conjunto, constituem essa filosofia de inovação na base da pirâmide. Inovar no 
mercado da BOP pode reverter o fluxo do conceito, das ideias e do método de gestão. Os 12 princípios estão elencados no Tabela 2.

Tabela 2

Doze Princípios de Prahalad

Desempenho de Preço Não só oferecer preços baixos, mas trabalhar com uma equilibrada relação de custos e benefícios dos produtos é desempenhar boas práticas de preço na BOP.

\begin{tabular}{ll}
\hline Inovações Híbridas & $\begin{array}{l}\text { Nem tecnologias avançadas com altos custos nem tecnologias obsoletas com } \\
\text { restrições, a hibridização de uma boa tecnologia com infraestrutura adequada é o } \\
\text { caminho para oferecer produtos inovadores na BOP. }\end{array}$ \\
\hline Escala das Operações & $\begin{array}{l}\text { Para atender às necessidades dos consumidores da BOP, é necessário focar nas } \\
\text { especificidades regionais. Em outras palavras, é necessário oferecer soluções locais } \\
\text { em mercados globais, assim, é possível inovar na BOP. }\end{array}$ \\
\hline $\begin{array}{l}\text { Desenvolvimento } \\
\text { Sustentável }\end{array}$ & $\begin{array}{l}\text { A revolução industrial que alavancou e fortaleceu os países ricos não se preocupou } \\
\text { com o gasto dos recursos naturais. Essa despreocupação está fora de qualquer agenda } \\
\text { política e empresarial nos dias de hoje. Portanto, para crescer na base da pirâmide, é } \\
\text { necessário produzir soluções sustentáveis e ecologicamente corretas. }\end{array}$
\end{tabular}

Identificação de

A funcionalidade dos produtos para a BOP não precisa ser altamente complexa e

Funcionalidade oferecer inúmeras soluções muitas vezes inutilizadas. Os consumidores da BOP buscam solucionar problemas de forma objetiva, ou seja, o produto para esse mercado tem que ser funcional e solucionar o problema do consumidor.

Inovação de Processos Tendo em vista as especificidades do mercado da BOP, é necessário adequar os processos para desenvolver inovações dentro da infraestrutura corrente. A inovação de processos é fundamental para fornecer produtos e serviços para a baixa renda.

Desespecialização do Oferecer produtos e serviços que sejam de fácil uso e não exijam especializações e

Trabalho

(Usabilidade) conhecimento avançado é fundamental para um consumidor da BOP. O conceito de plug and play ou o conceito de aprender a manusear os produtos e serviços de forma intuitiva e simples é fundamental para atuar na BOP.

Educação dos Clientes Além de o produto ser simples (desespecialização) e fácil de usar (usabilidade), é necessário que as empresas ensinem os clientes para que eles tenham domínio do que estão comprando. Essa prática facilita a adoção dos produtos na BOP.

Infraestrutura Hostil A maior parte da população da BOP reside em regiões com altas restrições de infraestrutura (saneamento, energia, água, transporte, educação, saúde etc.). Se atentar para essas restrições é fundamental para a difusão da inovação.

Interfaces

Ter interfaces amigáveis é fundamental, principalmente pelo fato de que muitas vezes é a primeira ocasião que um consumidor da BOP tem contato com produtos com atributos inovadores. Interfaces amigáveis proporcionam maior intenção para adoção de inovação.

Distribuição

Pela dificuldade de locomoção, nem sempre os consumidores podem ir até os lugares onde estão os bancos, hospitais, escolas e centros comerciais, por isso, inovar na distribuição para maior amplitude é fundamental para a adoção da inovação por parte da BOP.

Rompimento de

O sucesso na BOP desafia a sabedoria convencional, ou seja, para obter sucesso nesse Paradigmas mercado, lógicas contraintuitivas podem fazer mais sentido. O preconceito e o despreparo fazem com que a taxa de insucesso aumente. O mercado é grande e contém diversos subsegmentos, e essa heterogeneidade também exige uma quebra de paradigma.

Nota. Fonte: Elaborado pelos autores com base em Prahalad, C. K. (2005). The fortune at the bottom of the pyramid: Eradicating poverty through profits (pp.25-27). Upper Saddle River, NJ: Wharton School Publishing. 
Portanto, tendo em vista esses 12 princípios para inovação que as empresas precisam para proporcionar ao consumidor da BOP maior probabilidade de sua adoção, nossa proposição 4, envolvendo esses princípios como variáveis influenciadoras, aponta que:

Proposição 4. A relação entre as restrições da BOP e a adoção da inovação é positivamente influenciada pelos doze Princípios da Inovação de Prahalad (2005) - (P4a) Preço, (P4b) Inovações Híbridas, (P4c) Economia de Escala, (P4d) Sustentabilidade, (P4e) Funcionalidade, (P4f) Processos, (P4g) Usabilidade, (P4h), Educação (P4i) Infraestrutura, (P4j) Interface, (P4k) Distribuição e (P4l) Paradigmas.

\section{Inovação disruptiva}

A inovação disruptiva está relacionada às inovações para produtos existentes, mas com atributos sendo avaliados de maneira diferente, principalmente por consumidores não tradicionais, e muda as práticas sociais, o modo de viver, de trabalhar, de se relacionar (Christensen, 2001). Não é a tecnologia em si que importa, mas a tecnologia em uso. Essas inovações são posicionadas inicialmente para um público diferente daquele que costumeiramente é o alvo das inovações (sustentadoras). A inovação disruptiva começa com um público menos exigente e ganha força aos poucos até que passa a atender às necessidades também dos clientes mais exigentes, aí então passa a ser uma ameaça para as grandes empresas que se baseiam nas inovações sustentadoras (Corsi \& Di Minin, 2014).

Essas inovações são inicialmente de menor desempenho em relação aos principais atributos das tecnologias sustentadoras. Quando essas tecnologias atingem o mesmo desempenho que as tecnologias sustentadoras, elas começam o processo de disrupção, incomodando e ameaçando as empresas estabelecidas no mercado. Seus principais atributos são preço baixo, simplicidade, tamanho reduzido e, mais convenientes para o uso, sendo então bem posicionadas para o mercado da base da pirâmide (Corsi \& Di Minin, 2014).

As inovações disruptivas podem ser primeiramente comercializadas em mercados emergentes, pois suas características não agradam aos consumidores dos mercados tradicionais, ou das classes abastadas. Portanto, a área de Marketing tem mais responsabilidade sobre as tecnologias disruptivas do que o próprio setor de Tecnologia e P\&D. Assim, as inovações sustentadoras são posicionadas para a top of pyramid (TOP), e as inovações disruptivas são posicionadas para a BOP (Christensen, Johnson, $\&$ Rigby, 2002). Contudo, para atingir a lucratividade, a tecnologia disruptiva deve ser comercializada em grande escala, como qualquer outro mercado que trabalhe com baixas margens. A principal essência da inovação disruptiva é a capacidade de poder causar o fracasso de empresas grandes e estabelecidas em mercados desenvolvidos, por isso o nome disruptiva (Ray \& Ray, 2011).

A ideia de Christensen (2001) não era de mostrar como inovar em mercados emergentes e ganhar competitividade entre as grandes empresas, ele queria sinalizar para as grandes e estabelecidas empresas como elas poderiam ir à falência se não cuidassem dos pequenos concorrentes que têm potencial para crescimento, ou seja, a lógica é inversa. O autor se preocupava em se defender das pequenas e potenciais empresas, mas o objetivo aqui é também apontar que há uma forma de crescer no mercado e competir com grandes e estabelecidas empresas oferecendo produtos simples e baratos para um segmento que ainda é mal explorado.

Dadas as características da inovação disruptiva e as demandas dos consumidores que a inovação disruptiva pretende atingir, esta literatura é apropriada para estudar o mercado da BOP (Hart \& Christensen, 2002; Nogami \& Veloso, 2017). Os consumidores da BOP são os principais alvos da tecnologia disruptiva, porém, a inovação não deve ser disruptiva sem mudanças no modelo dos negócios das empresas (Pels \& Sheth, 2017). Para obter sucesso na baixa renda, é preciso inovação disruptiva no processo, na cadeia de distribuição, na gestão da organização, na educação dos consumidores, na forma de pagamento e nas pessoas envolvidas no negócio (Corsi \& Di Minin, 2014; Hart \& Christensen, 2002).

Portanto, considerando que o conceito de inovação disruptiva apresentado tem propriedades que se enquadram no mercado de baixa renda mesmo não sendo desenvolvidas com esse intuito, suas 
características tanto nos atributos do produto quanto nos cenários do mercado (consumidores e competidores) podem proporcionar ao consumidor da BOP maior adoção da inovação, conforme propomos na proposição 5 .

Proposição 5. A relação entre as restrições da BOP e a adoção da inovação é positivamente influenciada pelas características da Inovação Disruptiva - (P5a) Preço Baixo, (P5b) Simplicidade, (P5c) Tamanho Reduzido e (P5d) Conveniência de Uso.

\section{Inovação reversa}

Outro conceito de inovação adequado ao mercado da base da pirâmide é o da inovação reversa. Immelt, Govindarajan e Trimble (2009) mostram como a General Electric se beneficiou da presença em mercados emergentes, especificamente na China e na Índia, para desenvolver inovações que são introduzidas e comercializadas com sucesso inicialmente nos próprios mercados emergentes e depois chegam a ter penetração também em economias desenvolvidas, essa é a ideia principal da inovação reversa (Corsi \& Di Minin, 2014; Govindarajan \& Trimble, 2012; Immelt, Govindarajan, \& Trimble, 2009).

A inovação reversa é definida como uma inovação que tem maior probabilidade de ser adotada primeiramente em economias em desenvolvimento e posteriormente é adotada em economias desenvolvidas (Govindarajan \& Trimble, 2013). O conceito de inovação reversa foi construído tendo como premissa que inovar nos mercados emergentes não é simplesmente exportar produtos de países ricos com ajustes e adaptações para os países pobres, a ideia é começar do zero e desenvolver um produto específico para o mercado emergente tendo em vista suas necessidades e conjunturas regionais (Govindarajan \& Ramamurti, 2011; Brem \& Ivens, 2013).

Esse conceito foi criado porque, historicamente, as inovações são inicialmente criadas e adotadas em países ricos, contudo, o caminho inverso tem ocorrido, principalmente depois do processo de globalização e abertura dos mercados, e da crise de 2008, que refletiu em dificuldades financeiras com maior intensidade nos países desenvolvidos (Subramaniam et al., 2015). Uma vez que o mercado de alta renda se torna saturado, as grandes empresas começam a olhar para o mercado da base da pirâmide com outros olhos, então inovar nesse mercado se torna fundamental, contudo, a inovação nesse caso nem sempre acontece tal qual nos países ricos, por isso o conceito de inovação reversa ganha destaque (Brem \& Wolfram, 2014, Govindarajan \& Ramamurti, 2011).

Historicamente, as inovações reversas são muito raras de acontecer, pois, quando se pensa intuitivamente em inovação, a lógica é que ela seja de cima para baixo, ou dos países ricos para os países emergentes. Portanto, uma inovação criada nos países em desenvolvimento, com tecnologia local e depois exportada, e ainda, adotada pelos países desenvolvidos, parece mesmo um contrassenso. Tendo em vista essa premissa, a estratégia de glocalização faz mais sentido. Essa estratégia nada mais é do que a prática de exportação de produtos desenvolvidos em países ricos com ligeiras modificações para adequação ao mercado emergente, tornando-os mais baratos e com menos atributos.

A glocalização refere-se à presença da dimensão local na produção de uma cultura global (Immelt et al., 2009) e postula que o trabalho de inovação já ocorreu. As empresas podem atingir os mercados emergentes exportando versões ligeiramente modificadas de produtos globais desenvolvidos para clientes do mundo rico - em especial os modelos mais baratos e com menos recursos (Govindarajan \& Ramamurti, 2011). Porém, as premissas estão erradas (Govindarajan \& Trimble, 2013). Aquilo que funciona no mundo rico não vai automaticamente alcançar aceitação nos mercados emergentes, onde as necessidades dos clientes são completamente diferentes (Govindarajan \& Trimble, 2013). Em consequência, a inovação reversa está rapidamente ganhando força, e essa situação tende a aumentar.

Dessa forma, a inovação reversa tem um papel não só econômico, mas também social muito importante, que é o de redistribuição da renda, do acesso à tecnologia e da riqueza dos países e empresas (Immelt et al., 2009). Com base na inovação reversa, os países em desenvolvimento podem acelerar o 
crescimento econômico e consequentemente contribuir para outras grandes áreas como: comunicações, infraestrutura, educação, ciência e saúde.

Tendo em vista as premissas apresentadas, podemos citar as quatro fases que descrevem a inovação reversa. As duas primeiras são chamadas de fase da globalização ou da glocalização, pois se utilizam da abertura de mercado para privilegiar o próprio mercado. Nessas duas fases iniciais, os produtos são produzidos em países desenvolvidos e vendidos para os mercados emergentes, depois esses produtos são produzidos nos mercados emergentes para os próprios consumidores desses países, porém a maior parte do lucro retorna para as empresas dos países desenvolvidos.

A inovação reversa se inicia então, efetivamente, na fase três, quando os produtos começam a ter características locais dos mercados emergentes (Govindarajan \& Trimble, 2013). Em outras palavras, começa-se a levar em consideração as premissas descritas e analisar as defasagens entre as economias desenvolvidas e as economias em desenvolvimento para criação e lançamento de novos produtos. Por fim, a inovação reversa se completa quando os produtos produzidos nos mercados emergentes com características locais são vendidos para todo o mundo, ficando o lucro para as empresas desses países. Essa é a quarta e última fase, que é muito difícil de acontecer. É a inovação reversa efetiva, que tem por origem as características emergentes e conquista os mercados mais desenvolvidos (Govindarajan \& Ramamurti, 2011).

A inovação reversa compartilha algumas semelhanças com a inovação disruptiva. A inovação reversa também é diferente da inovação radical e da inovação com características de destruição criativa, bem como é diferente da concepção de inovação disruptiva (Govindarajan \& Trimble, 2013). Embora tenham características parecidas, a inovação disruptiva e a inovação reversa apresentam diferenças. A principal semelhança é que ambas são desenvolvidas inicialmente para consumidores de baixa renda e, posteriormente, atingem os consumidores com maior renda (Govindarajan \& Ramamurti, 2011). Outra semelhança é que os dois tipos de inovação transformam empresas e economias com pouca expressão em empresas e economias com alta competitividade no mercado, preocupando as grandes empresas e as economias desenvolvidas. Ainda, ambos os conceitos são propostos como uma forma prescritiva para as empresas atuarem no mercado, da BOP ou não - middlevel, fortalecendo a revisão da literatura que é escassa do ponto de vista do consumidor - microlevel (Viswanathan et al., 2011).

Considerando a literatura sobre inovação reversa, suas características podem proporcionar ao consumidor da BOP maior adoção de inovação, conforme proposição 6.

Proposição 6. A relação entre as restrições da BOP e a adoção da inovação é positivamente influenciada pelas características da Inovação Reversa - (P6a) Desenvolvida em Mercados Emergentes, (P6b) Características Regionais, (P6c) Menor Desempenho e (P6d) Sustentabilidade.

\section{Inovação inclusiva}

O recente interesse na inovação para atender os mercados da BOP produziu até agora relativamente poucas evidências sobre o papel da política (Foster \& Heeks, 2013a). Nesse contexto, a inovação inclusiva foi construída em cima de um círculo de reforço da inovação adaptativa, a concorrência dinâmica e presença de intermediários de inovação dentro das comunidades pobres para promover a inovação na BOP de maneira mais eficaz (Foster \& Heeks, 2013b). Diferentemente dos conceitos apresentados até agora pela literatura norte-americana e por autores norte-americanos (Anderson \& Billou, 2007), além dos indianos que se encontram nas universidades norte-americanas (Govindarajan \& Ramamurti, 2011; Govindarajan \& Trimble, 2012; Prahalad, 2012; Venkatesh, Thong, \& Xu, 2012), o conceito de inovação inclusiva surgiu na Inglaterra, cenário europeu, proposto por Christopher Foster e Richard Heeks em 2013a.

Foster e Heeks (2013b) definem a inovação inclusiva como a inclusão de alguns aspectos inovadores de grupos que são marginalizados. Como Heeks, Foster e Nugroho (2014) apontam, essa definição leva em consideração duas questões: quem são os marginalizados e como se caracterizam suas necessidades de serem incluídos no contexto da inovação. Grupos identificados como marginalizados, 
de forma geral, incluem mulheres, jovens, pessoas com deficiência, minorias étnicas e empreendedores informais (Heeks, Foster, \& Nugroho, 2014). Mas o foco principal tem sido as pessoas de baixa renda, aqueles que ganham US\$1,25 ou US\$2,00 ou até US\$2,50 por dia.

A necessidade de inclusão no contexto da inovação tem duas principais abordagens: a inclusão do processo (inclusivity of process) e a inclusão de saída (inclusivity of output). A primeira é a participação e o envolvimento dos consumidores de baixa renda no desenvolvimento e design dos produtos e estruturas de mercados. A segunda são os meios pelos quais a inovação será criada, desenvolvida, difundida e adotada pelos indivíduos. Ambas as etapas são altamente dependentes da participação dos consumidores (microlevel).

A relação consumidor-fabricante sempre foi fundamental no processo de inovação, no mercado de baixa renda onde existem diversas especificidades. Essa relação deve ser intensificada, contudo não é isso que tem acontecido. Outro fator são as restrições de infraestrutura e escassez de recursos da BOP, que os tornam com mais aversão a riscos e consequentemente há maior aversão em adotar inovação. A heterogeneidade desse segmento também caracteriza o mercado como mais complexo para ser trabalhado. Todos esses elementos já foram citados anteriormente por autores diferentes em contextos distintos (Foster \& Heeks, 2013b).

Sobre as características do produto, para a inovação inclusiva, os atributos, desempenho e qualidade também devem ser inferiores em relação à tecnologia mais atual até um ponto ótimo aceitável para a BOP que balanceie a relação preço/desempenho. Também devem ser desenvolvidos produtos específicos para esse mercado a partir do zero, em vez de redução de atributos e adaptações geralmente limitadas (Heeks et al., 2014).

Nessa fase inicial, a literatura sobre inovação inclusiva pode ser dividida em três categorias: descritiva, analítica e prescritiva (Heeks et al., 2014). Os textos descritivos apenas apresentam exemplos de inovação inclusiva; os analíticos investigam o conceito de um ponto de vista conceitual e acadêmico; os prescritivos fornecem direcionamentos para práticas relacionadas à inovação inclusiva. Heeks et al. (2014) defendem e estimulam a produção de conhecimento, ou seja, perspectiva analítica, para avançar a literatura sobre o assunto e então gerar mais e melhores textos descritivos e prescritivos. Até então, a inovação inclusiva tem seis níveis.

Primeiro, a intenção, ou seja, uma inovação é inclusiva se a sua intenção é de que a inovação consiste em atender necessidades, desejos ou problemas dos grupos marginalizados. Segundo, o consumo, isto é, uma inovação é inclusiva se for aprovada e utilizada pelo grupo marginalizado. Terceiro, o impacto, quer dizer, uma inovação é inclusiva, se ela tem um impacto positivo sobre as condições de vida do grupo marginalizado. Quarto, um processo, ou melhor, uma inovação é inclusiva se o grupo marginalizado estiver envolvido no desenvolvimento da inovação. Quinto, a estrutura, ou seja, uma inovação é inclusiva se for criada dentro de uma estrutura que é, em si, inclusiva. Sexto, pósEstrutura, isto é, uma inovação é inclusiva se ela é criada dentro de um quadro de conhecimento e de discurso que são inclusivos.

Os níveis têm uma relação hierárquica, pois cada nível envolve um aprofundamento gradual e ampliação da extensão da inclusão do grupo excluído em relação à inovação. Em geral, cada nível aceita a inclusão dos níveis abaixo, mas empurra a extensão da inclusão adicional (Foster, 2014). Não há nível certo ou errado, é preciso reconhecer em qualquer texto sobre inovação inclusiva seja ela uma política, declaração pública, ou artigo científico, qual grupo e que tipo de inclusão está subjacente a esse texto (Foster \& Heeks, 2014).

Considerando a literatura sobre inovação inclusiva, suas características podem proporcionar ao consumidor da BOP maior adoção da inovação, conforme propomos na proposição 7 .

Proposição 7. A relação entre as restrições da BOP e a adoção da inovação é positivamente influenciada pelas características da Inovação Inclusiva - (P7a) Intenção, (P7b) Consumo, (P7c) Impacto, (P7d) Processo, (P7e) Estrutura e (P7f) Pós-Estrutura. 


\section{Framework Conceitual}

Finalmente, depois da revisão da literatura, é possível apresentar o framework conceitual do artigo, que teve como principal inspiração acadêmica o modelo de Nakata e Weidner (2012) e foi construído a partir da revisão da literatura acerca da inovação e mercado de baixa renda. Enquanto Nakata e Weidener (2012) destacam os elementos do composto de Marketing e o contexto social como variáveis influenciadoras, o presente framework se fundamenta na literatura sobre inovação e mercado da base da pirâmide.

Em nosso framework está presente a relação direta entre a variável independente Restrições da BOP e a variável dependente Adoção da Inovação. A primeira proposição é que as restrições da BOP têm uma relação negativa com a adoção da inovação. Então, para identificar maneiras de reduzir esse efeito e potencializar a adoção da inovação, levantamos seis conceitos na fundamentação teórica com a proposta de assumirem papéis influenciadores nessa relação, que são as proposições de 2 a 7 , conforme Figura 1.

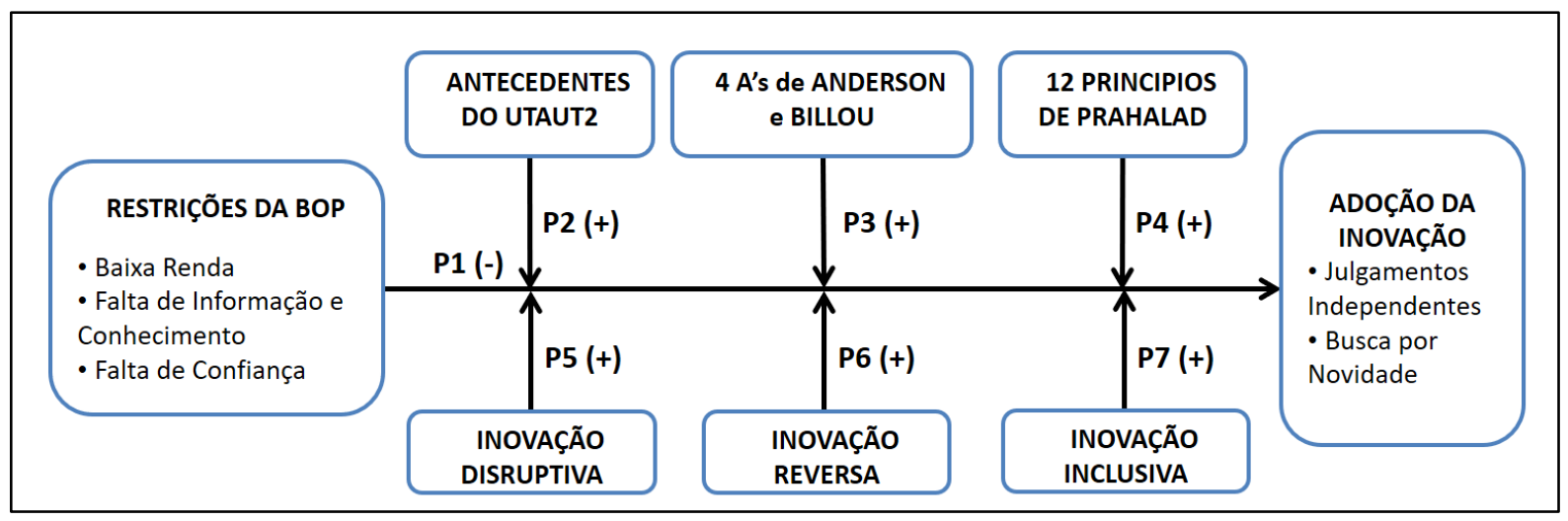

Figura 1. Framework Conceitual

Fonte: Elaborada pelos autores.

A Figura 1 é a representação de todas as proposições juntas em um único framework. Para uma possível investigação empírica que possa ser realizada em pesquisas futuras, recomendamos que seja construído um modelo estatístico com base nesse framework, no qual as variáveis influenciadoras possam agir como variáveis moderadoras do efeito principal.

Para tanto, essas influências devem ser analisadas separadamente para que não haja muitas covariáveis na relação direta entre variável independente e variável dependente. Ademais, muitos itens dentro de cada constructo são sobrepostos, por exemplo, o item preço que está presente em quase todos os casos. Do ponto de vista de um modelo estrutural, são necessárias análises e revisões para identificar os melhores indicadores (variáveis observáveis) para verificação empírica.

Do ponto de vista gerencial, a discussão inicia na variável independente, que são as restrições. Para aumentar a taxa de adoção da inovação, é necessário partir do pressuposto de que os consumidores de baixa renda enfrentam dificuldades em várias facetas do cotidiano que consumidores de alta renda, que estão acostumados a adotar inovação mais facilmente, não enfrentam. Complementarmente, o que pode estimular a adoção da inovação são esses fatores, já revisados na literatura, que podem alterar o efeito dessa relação, estimulando a taxa de adoção da inovação.

Ademais, reforça-se a ideia de que as tradicionais decisões de marketing para atuar no mercado de baixa renda não resultam no mesmo efeito quando é direcionada ao mercado de alta renda, que está mais acostumado a adotar produtos e serviços com tecnologias mais avançadas. É preciso partir do pressuposto de que existem restrições nesse contexto, assim, é possível entender as dificuldades e as incertezas que influenciam a adoção da inovação. Diminuindo a aversão ao risco, falta de conhecimento, 
falta de confiança e propondo melhores condições de uso, é possível que o consumidor de baixa renda tenha maior propensão a adotar inovações que costumeiramente não adota.

Esse framework se adapta para analisar a adoção da inovação em ambientes de incerteza, como países com alto índice de pobreza, favelas em grandes metrópoles, vilarejos rurais onde há fraca infraestrutura, acampamentos de refugiados em que as pessoas passaram por um grande trauma social e individual, locais onde aconteceram desastres naturais e outros lugares nos quais as restrições das pessoas sejam muito evidentes, afetando a adoção da inovação. O framework tem como principal contribuição analisar de que forma a relação negativa entre essas restrições e a adoção da inovação podem ser alteradas, ou seja, influenciadas pelas variáveis apresentadas neste estudo.

\section{Considerações Finais}

Pesquisas sobre inovação e mercado de baixa renda focam eminentemente no desenvolvimento de produtos e gestão de marketing (Nakata \& Viswanathan, 2012). Essa prática resulta em textos prescritivos sobre gestão de empresas multinacionais, tanto em mercados desenvolvidos quanto em mercados emergentes. Do ponto de vista do indivíduo, tem sido dada pouca atenção para compreender a adoção da inovação pelos consumidores de baixa renda. O presente artigo buscou investigar o conceito de inovação por essa perspectiva bottom-up, focando principalmente nos consumidores.

A principal contribuição do presente estudo está na proposta de um framework teórico que busca demonstrar como algumas variáveis podem influenciar positivamente uma relação negativa que pressupõe baixa taxa de adoção da inovação. Nessa contribuição, não negligenciamos as restrições encontradas no mercado de baixa renda, conforme aponta Mendoza (2011), ao destacar o conceito de poverty penalty, e Khanna e Palepu (2013), ao destacar as barreiras estruturais no contexto da BOP. Enquanto muitos modelos buscam identificar os fatores positivos para estimular a adoção da inovação, o presente framework aborda um efeito negativo.

Finalizamos o texto deixando como sugestão para pesquisas futuras a verificação empírica do framework proposto. Para certos constructos, já existem algumas escalas desenvolvidas para serem aproveitadas ou adaptadas. Para outros, o desenvolvimento e a validação dos constructos são necessários. Ademais, alertamos para a possível sobreposição de indicadores que pode existir dentro dos 6 conceitos revisados. Uma vez que são conceitos interdependentes, alguns elementos podem estar presentes em mais de um constructo, podendo causar diferentes tipos de interferência e problemas de erros do ponto de vista estatístico.

\section{Contribuições}

$1^{\circ}$ Autor: Introdução, Fundamentação Teórica, Framework Conceitual e Considerações Finais.

$2^{\circ}$ Autor: Introdução, Fundamentação Teórica, Framework Conceitual e Considerações Finais.

\section{Referências}

Abdelnour, S., \& Branzei, O. (2010). Fuel-efficient stoves for darfur: The social construction of subsistence marketplaces in post-conflict settings. Journal of Business Research, 63(6), 617-629. https://doi.org/10.1016/j.jbusres.2009.04.027

Anderson, J., \& Billou, N. (2007). Serving the world's poor: Innovation at the base of the economic $\begin{array}{llll}\text { pyramid. Journal of Business } & \text { Strategy, }\end{array}$ 
https://doi.org/10.1108/02756660710732611

Anderson, J., \& Markides, C. (2007). Strategic innovation at the base of the economic pyramid. MIT Sloan Management Review, 49(1), 83-88. https://sloanreview.mit.edu/article/strategicinnovation-at-the-base-of-the-pyramid/

Barki, E., \& Parente, J. (2010). Consumer behaviour of the base of the pyramid market in Brazil. Greener Management International, 11-23. https://doi.org/10.9774/GLEAF.3062.2006.wi.00004

Brem, A., \& Ivens, B. (2013). Do frugal and reverse innovation foster sustainability? Introduction of a conceptual framework. Journal of Technology Management for Growing Economies, 4(2), 3150. https://doi.org/10.15415/jtmge.2013.42006

Brem, A., \& Wolfram, P. (2014). Research and development from the bottom up - introduction of terminologies for new product development in emerging markets. Journal of Innovation and Entrepreneurship, 3(1), 1-22. https://doi.org/10.1186/2192-5372-3-9

Christensen, C. (2001). The past and future of competitive advantage. Sloan Management Review, 42(2), 105-109. https://sloanreview.mit.edu/article/the-past-and-future-of-competitive-advantage/

Christensen, C., Johnson, M. W., \& Rigby, D. K. (2002). Foundations for growth: How to identify and build disruptive new businesses. MIT Sloan Management Review, 43(3), 22-32. https://sloanreview.mit.edu/article/foundations-for-growth-how-to-identify-and-builddisruptive-new-businesses/

Corsi, S., \& Di Minin, A. (2014). Disruptive innovation in reverse: Adding a geographical dimension to disruptive innovation theory. Creativity and Innovation Management, 23(1), 76-90. https://doi.org/10.1111/caim.12043

Davis, F. D., Bagozzi, R. P., \& Warshaw, P. R. (1989). User acceptance of computer technology: A comparison of two theoretical models. Management Science, 35(8), 982-1003. https://doi.org/10.1287/mnsc.35.8.982

Faria, A., \& Hemais, M. (2017). Rethinking the bottom of the pyramid: A critical perspective from an emerging economy. Marketing Theory, 17(3), 271-287. https://doi.org/10.1177/1470593117704283

Fishbein, M., \& Ajzen, I. (1975). Belief, attitude, intention and behavior: An introduction to theory and research. Reading, MA: Addison-Wesley Pub. Co.

Foster, C. (2014). Does quality matter for innovations in low income markets? The case of the kenyan mobile phone sector. Technology in Society, 38, 119-129. https://doi.org/10.1016/j.techsoc.2014.03.003

Foster, C., \& Heeks, R. (2013a). Analyzing policy for inclusive innovation: the mobile sector and baseof-the-pyramid markets in kenya. Innovation and Development, 3(1), 103-119. https://doi.org/10.1080/2157930X.2013.764628

Foster, C., \& Heeks, R. (2013b). Conceptualising inclusive innovation: Modifying systems of innovation frameworks to understand diffusion of new technology to low-income consumers. European Journal of Development Research, 25(3), 333-355. https://doi.org/10.1057/ejdr.2013.7

Foster, C., \& Heeks, R. (2014). Nurturing user-producer interaction: Inclusive innovation flows in a low-income mobile phone market. Innovation and Development, 4(2), 221-237. https://doi.org/10.1080/2157930X.2014.921353

Govindarajan, V., \& Ramamurti, R. (2011). Reeverse innovation, emerging markets, and global 
strategy. Global Strategy Journal, 1(3/4), 191-205. https://doi.org/10.1002/gsj.23

Govindarajan, V., \& Trimble, C. (2012). Reverse innovation: A global growth strategy that could preempt disruption at home. Strategy \& Leadership, 40(5), 5-11. https://doi.org/10.1108/10878571211257122

Govindarajan, V., \& Trimble, C. (2013). Reverse innovation: Create far from home, win everywhere. Boston, MA: Harvard Business Press.

Hammond, A. L., Kramer, W. J., Katz, R. S., Tran, J. T., \& Walker, C. (2007). The next 4 billion: Market size and business strategy at the base of the pyramid. Retrieved from https://www.ifc.org/wps/wcm/connect/3c2787004cc75e6094d7b59ec86113d5/Pub_009_The\%2 BNext2B4\%2BBillion.pdf?MOD=AJPERES

Hang, C., Chen, J., \& Subramian, A. M. (2010). Developing disruptive products for emerging economies: Lessons from asian cases. Research-Technology Management, 53(4), 21-26. https://doi.org/10.1080/08956308.2010.11657637

Hart, S., \& Christensen, C. (2002). The great leap: Driving innovation from the base of the pyramid. Sloan Management Review, 44(1), 51-56. https://sloanreview.mit.edu/article/the-great-leapdriving-innovation-from-the-base-of-the-pyramid/

Heeks, R., Foster, C., \& Nugroho, Y. (2014). New models of inclusive innovation for development. Innovation and Development, 4(2), 175-185. https://doi.org/10.1080/2157930X.2014.928982

Hemais, M. W., Borelli, F. C., Casotti, L. M., \& Dias, P. I. R. (2014). Economia, marketing e indivíduos de baixa renda: O interesse após uma história de indiferença. Revista de Ciências da Administração, 16(39), 49-64. https://doi.org/10.5007/2175-8077.2014v16n39p49

Hirschman, E. C. (1980). Innovativeness, novelty seeking, and consumer creativity. Journal of Consumer Research, 7(3), 283-295. https://doi.org/10.1086/208816

Immelt, J. R., Govindarajan, V., \& Trimble, C. (2009). How GE is disrupting itself. Harvard Business Review, 87(10), 56-65. https://hbr.org/2009/10/how-ge-is-disrupting-itself

Kaplinsky, R., Chataway, J., Clark, N., Hanlin, R., Kale, D., Muraguri, L., \& Wamae, W. (2009). Below the radar: What does innovation in emerging economies have to offer other low-income economies? International Journal of Technology Management and Sustainable Development, 8(3), 177-197. https://doi.org/10.1386/ijtm.8.3.177/1

Khanna, T., \& Palepu, K. (2013). Winning in emerging markets: A road map for strategy and execution. Boston, MA: Harvard Business Press. https://doi.org/10.1080/1051712X.2012.666487

Kolk, J. E. M.., Rivera-Santos, M., \& Rufin, C. (2014). Reviewing a decade of research on the "Base/Bottom of the Pyramid"(BOP) concept. Business \& Society, 53(3), 338-377. https://doi.org/10.1177/0007650312474928

Manning, K. C., Bearden, W. O., \& Madden, T. J. (1995). Consumer innovativeness and the adoption $\begin{array}{llll}\text { process. Journal of Consumer } & \text { 329-345. }\end{array}$ https://doi.org/10.1207/s15327663jcp0404_02

Mendoza, R. U. (2011). Why do the poor pay more? Exploring the poverty penalty concept. Journal of International Development, 23(1), 1-28. https://doi.org/10.1002/jid.1504

Midgley, D. F., \& Dowling, G. R. (1978). Innovativeness: The concept and its measurement. Journal of Consumer Research, 4(4), 229-242. https://doi.org/10.1086/208701

Nakata, C., \& Viswanathan, M. (2012). From impactful research to sustainable innovations for 
subsistence marketplaces. Journal of Business Research, 65(12), 1655-1657. https://doi.org/10.1016/j.jbusres.2012.02.005

Nakata, C., \& Weidner, K. (2012). Enhancing new product adoption at the base of the pyramid: A contextualized model. Journal of Product Innovation Management, 29(1), 21-32. https://doi.org/10.1111/j.1540-5885.2011.00876.x

Nogami, V. K. C., Carvalho, D. D., Toledo, G. L., Veloso, A. R., \& Gouvêa, M. A. (2015). Innovation intensity and adoption at the base of the pyramid market: A study of household appliances. Future Studies Research Journal: Trend and Strategies, 7(1), 33-56. https://doi.org/10.24023/FutureJournal/2175-5825/2015.v7i1.179

Nogami, V. K. C., \& Veloso, A. R. (2017). Disruptive innovation in low-income contexts: Challenges and state-of-the-art national research in marketing. Innovation \& Management Review, 14(2), 162-167. https://doi.org/10.1016/j.rai.2017.03.005

Nogami, V. K. C., Vieira, F. G. D., \& Veloso, A. R. (2018). Concept of innovation in low-income $\begin{array}{llll}\text { market. Review of Business } & \text { Management, 20(1), }\end{array}$ https://doi.org/10.7819/rbgn.v20i1.3044

Parente, J., Limeira, T. M., \& Barki, E. (2008). Varejo para a baixa renda. Porto Alegre: Bookman.

Pels, J., \& Sheth, J. N. (2017). Business models to serve low-income consumers in emerging markets. Marketing Theory, 17(3), 373-391. https://doi.org/10.1177/1470593117704262

Prahalad, C. K. (2005). The fortune at the bottom of the pyramid: Eradicating poverty through profits. Upper saddle river, NJ: Wharton School Publishing.

Prahalad, C. K. (2012). Bottom of the pyramid as a source of breakthrough innovations. Journal of Product Innovation Management, 29(1), 6-12. https://doi.org/10.1111/j.1540-5885.2011.00874.x

Ramani, S. V., \& Mukherjee, V. (2014). Can breakthrough innovations serve the poor (BOP) and create reputational (CSR) value? Indian case studies. Technovation, 34(5), 295-305. https://doi.org/10.1016/j.technovation.2013.07.001

Ray, S., \& Ray, P. K. (2011). Product innovation for the people's car in an emerging economy. Technovation, 31(5), 216-227. https://doi.org/10.1016/j.technovation.2011.01.004

Subramaniam, M., Ernst, H., \& Dubiel, A. (2015). From the special issue editors: Innovations for and from emerging markets. Journal of Product Innovation Management, 32(1), 5-11. https://doi.org/10.1111/jpim.12167

Venkatesh, V., Morris, M. G., Davis, G. B., \& Davis, F. D. (2003). User acceptance of Information technology: Toward a unified view. MIS Quarterly, 27(3), 425-478. https://doi.org/10.2307/30036540

Venkatesh, V., Thong, J. Y., \& Xu, X. (2012). Consumer acceptance and use of information technology: Extending the unified theory of acceptance and use of technology. MIS Quarterly, 36(1), 157178. https://doi.org/10.2307/41410412

Venugopal, S., \& Viswanathan, M. (2017). The subsistence marketplaces approach to poverty: Implications for marketing theory. Marketing Theory, 17(3), 341-356. https://doi.org/10.1177/1470593117704282

Viswanathan, M. (2016). Bottom-up enterprise insights from subsistence marketplaces. UrbanaChampaign, IL: Madhu Viswanathan.

Viswanathan, M., \& Rosa, J. A. (2010). Understanding subsistence marketplaces: Toward sustainable 
consumption and commerce for a better world. Journal of Business Research, 63(6), 535-537. https://doi.org/10.1016/j.jbusres.2009.06.001

Viswanathan, M., Shultz, C. J., \& Sridharan, S. (2014). Introduction to the special issue on subsistence marketplaces: From micro-level insights to macro-level impact. Journal of Macromarketing, 34(2), 119-121. https://doi.org/10.1177/0276146714522266

Viswanathan, M., \& Sridharan, S. (2012). Product development for the BOP: Insights on concept and prototype development from university-based student projects in India. Journal of Product Innovation Management, 29(1), 52-69. https://doi.org/10.1111/j.1540-5885.2011.00878.x

Viswanathan, M., Yassine, A., \& Clarke, J. (2011). Sustainable product and market development for subsistence marketplaces: Creating educational initiatives in radically different contexts. Journal of Product Innovation Management, 28(4), 558-569. https://doi.org/10.1111/j.15405885.2011.00825.x

Weidner, K. L., Rosa, J. A., \& Viswanathan, M. (2010). Marketing to subsistence consumers: lessons from practice. Journal of Business Research, 63(6), 559-569. https://doi.org/10.1016/j.jbusres.2009.02.022

Yurdakul, D., Atik, D., \& Dholakia, N. (2017). Redefining the bottom of the pyramid from a marketing perspective. Marketing Theory, 17(3), 289-303. https://doi.org/10.1177/1470593117704265

\section{Dados dos Autores}

Vitor Koki da Costa Nogami

Av. Colombo 5.790, 87020-900, Maringá, PR, Brasil.

E-mail: vitornogami@gmail.com, vkcnogami2@uem.br

Andres Rodriguez Veloso

Av. Prof. Luciano Gualberto, 908, Butantã, 05508-010, São Paulo, SP, Brasil.

E-mail: veloso@usp.br 